\title{
Do antihypertensive drugs precipitate diabetes in predisposed men?
}

\author{
Einar T Skarfors, Hans O Lithell, Ingemar Selinus, Hans Åberg
}

Abstract

Objective-To evaluate the influence of antihypertensive treatment and metabolic characteristics on the development of diabetes mellitus in middle aged men.

Design-Prospective study over an average of nine years.

Setting-Community based health survey of middle aged men carried out at the University of Uppsala.

Subjects-Seventy three hypertensive men aged 49-54 and 65 normotensive controls matched for body mass index, glucose disappearance rate ( $k$ value) at an intravenous glucose tolerance test, and serum triglyceride and cholesterol concentrations.

Interventions-Hypertensive group was treated with $\beta$ blockers, thiazides, hydralazine, or combinations of these drugs. Treatment was not randomised.

Measurements and main results-Intravenous glucose tolerance, fasting blood glucose and serum lipid and insulin concentrations, body weight and height, three skinfold measurements, and blood pressure were recorded both during an initial health screening survey in 1970-3 and at a follow up survey in 1980-3. In the period between the two surveys 12 hypertensive men and two controls developed diabetes. Review of values obtained at the initial survey showed that the hypertensive men who developed diabetes or impaired glucose tolerance could be distinguished from those hypertensive men who did not by virtue of a higher fasting serum insulin concentration $(26.1 v 15.2 \mathrm{mU} / \mathrm{l}$ (confidence interval of difference $-15 \cdot 2$ to $-6 \cdot 2)$ ), a lower peak serum insulin concentration $(78.9 v 94.3 \mathrm{mU} /$ (confidence interval of difference $-1 \cdot 1$ to $41 \cdot 1)$ ), and a lower $k$ value $(1.29 v 1.68$ (confidence interval of difference -0.02 to 0.68 )). The insulin index (peak insulin concentration divided by fasting insulin concentration), however, decreased significantly in the hypertensive men over time irrespective of whether they developed diabetes but did not change in the controls. Furthermore, the serum triglyceride concentration increased in the treated group and decreased in the controls.

Conclusion-A severalfold difference in the incidence of diabetes between treated hypertensive and non-treated, normotensive men may be a consequence of the treatment, which may be particularly deleterious in men predisposed to diabetes.

and Family Medicine,

Uppsala University,

Uppsala, Sweden

Einar T Skarfors, MD,

consultant

Hans O Lithell, MD, professor

Ingemar Selinus, $\mathrm{MD}$,

statistician

Hans Åberg, MD, professor

Correspondence and requests for reprints to: $\mathrm{Dr}$ Einar Skarfors, Department of Geriatrics, Uppsala University, PO Box 12042, S-750 12 Uppsala, Sweden. glucose tolerance (and the development of diabetes mellitus) in the two groups, who were metabolically similar at the start of the study. The hypertensive men received drug treatment throughout, whereas the

Introduction

Numerous reports have expressed concern about the habolic effects of antihypertensive drugs. may derange lipid metabolism or precipitate diabetes. We describe changes in carbohydrate metabolism and serum lipoprotein concentrations in hypertensive men after almost 10 years of treatment compared with a matched non-hypertensive control group. Our intenmatched non-hypertensive control group. Our inten- controls were normotensive at the start and not taking drugs at follow up.

\section{Subjects and methods}

The hypertensive and control groups were derived from a larger cohort of men who were participating in a health survey and who were examined in 1970-3 and again in 1980-3.

\section{HYPERTENSIVE GROUP}

The initial health survey was conducted among 2322 men born in 1920-4 and who therefore were aged 50 at the time of first examination. ${ }^{12}$ Of the 174 men identified who had primary hypertension (figure), 87 were newly diagnosed cases (untreated diastolic pressure $\geqslant 105 \mathrm{~mm} \mathrm{Hg}$ on two occasions) and 87 already receiving treatment. Treatment at a special hypertension clinic was offered to all these men, 91 of whom declined because they were attending other physicians. The remaining 83 men accepted and subsequently were checked twice a year for almost a decade. ${ }^{13}$ Three of the hypertensive men were known to be diabetic at the time of the first health survey and had blood glucose concentrations of $6 \cdot 7-9 \cdot 8 \mathrm{mmol} / \mathrm{l}$.

All men in the first health survey who had serum cholesterol or triglyceride concentrations, or both, above the 80th centile on two occasions were treated for hyperlipidaemia. Twenty three of the hypertensive men were given dietary advice for this reason. Pharmacological treatment of hypertension, however, was given priority over pharmacological treatment of hyperlipidaemia in the hyperlipidaemic hypertensives.

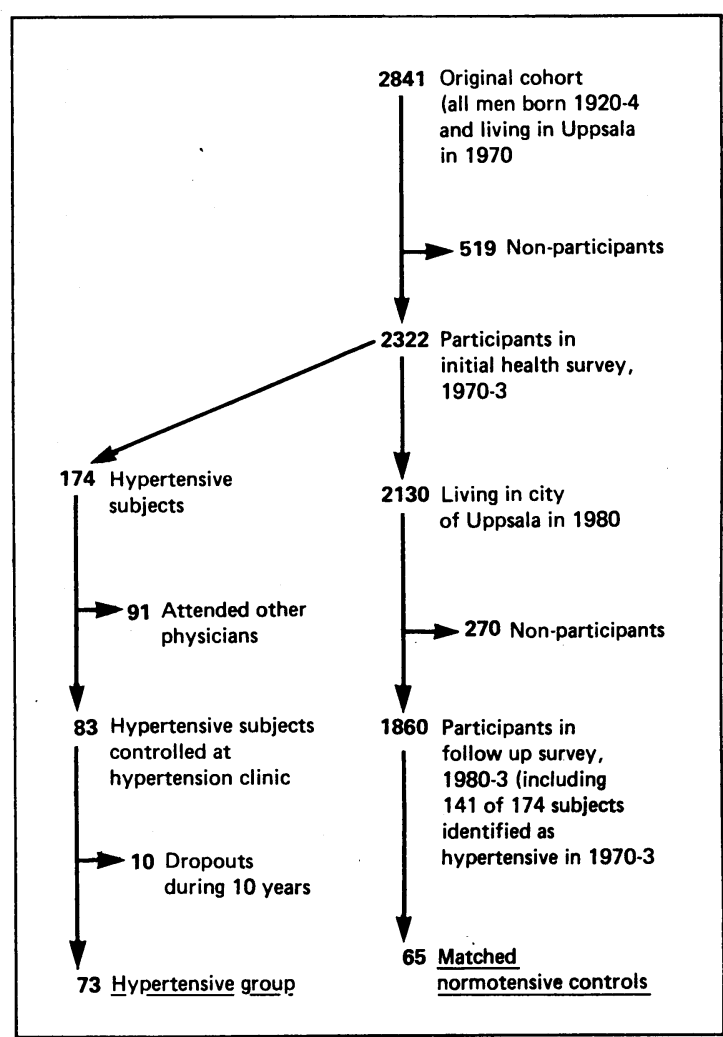

Flow diagram of composition of the two study groups of men 
The second health survey was carried out among all participants who were still living in Uppsala in 1980-3 and who attended for examination. They included 73 of the hypertensive follow up group.

\section{NORMOTENSIVE CONTROLS}

Controls were chosen from the non-hypertensive men examined in the initial survey (figure). As hypertensive people are more often obese than their normotensive peers and more commonly have impaired glucose tolerance ${ }^{14}$ and hyperlipidaemia, the control group was obtained by matching for body mass index, glucose disappearance rate ( $k$ value) at an intravenous glucose tolerance test (available in most cases), and serum triglyceride and cholesterol concentrations, these data having been obtained in the initial survey. Three controls were selected by computer for each hypertensive man. They were matched, firstly, for body mass index, then for serum triglyceride and cholesterol concentrations, and, finally, for $\mathbf{k}$ value from the intravenous glucose tolerance test. The controls were invited to attend in the order in which they were identified by the computer. If one did not attend the second was invited and so on. The matching procedure was performed before the start of the second health survey in 1980 .

At the second survey 65 normotensive controls were found to be suitable for the analysis.

\section{PROCEDURES}

For both the initial survey and the follow up survey subjects were contacted by letter and invited to attend for examination. They were instructed to fast and refrain from smoking from midnight. No dietary recommendations were given for the days preceding the examination

Intravenous glucose tolerance tests were performed during both examinations in most hypertensive and control men together with measurements of anthropometric variables, blood pressure, and serum lipid and lipoprotein concentrations. Blood pressure and anthropometric measurements were recorded as far as possible in the same way on the two occasions. Blood pressure was measured on the right arm after 10 minutes' recumbency by using a mercury manometer. Systolic and diastolic pressures were read to the nearest $5 \mathrm{~mm} \mathrm{Hg}$, diastolic pressure being recorded at Korotkoff phase V. Fatfold thickness was measured with a calliper ${ }^{15}$ on the back of the middle portion of the right upper arm, just below the scapula, and to the right of the umbilicus.

The intravenous glucose tolerance test was performed by the same method in the two surveys. At the first screening examination 1692 of the 2322 participants were tested. Glucose $0.5 \mathrm{~g} / \mathrm{kg}$ as a $50 \%$ solution was given into an antecubital vein over about two and a half minutes. Samples for blood glucose determination were drawn before and $20,30,40,50$, and 60 minutes after the start of the injection. The subjects remained supine throughout. Glucose tolerance was expressed as the $k$ value, as described. ${ }^{16}$

In addition, the fasting blood glucose concentration was measured in all subjects and an oral glucose tolerance test performed at follow up in those with values higher than $5.6 \mathrm{mmol} / \mathrm{l}$. A dose of $75 \mathrm{~g}$ glucose in $300 \mathrm{ml}$ water was given and blood samples for glucose assay taken before and $30,60,90$, and 120 minutes after intake. On the basis of the oral glucose tolerance test the subjects were divided into those with normal or impaired glucose tolerance and those with diabetes mellitus, as defined by the National Diabetes Data Group. ${ }^{17}$ In contrast with the recommendation of that group, however, only one oral glucose tolerance test was performed. The classification of diabetes at the first investigation was made in retrospect and thus was based on a fasting blood glucose concentration (available in all subjects) of $\geqslant 6.7 \mathrm{mmol} / \mathrm{l}$ either alone or in combination with a $k$ value of $\leqslant 0.9$ from the intravenous glucose tolerance test, which was performed in 54 of the 73 hypertensive men and 46 of the 65 controls.

\section{LABORATORY METHODS}

The method of measuring the blood glucose concentration differed between the two surveys. In the follow up survey glucose was measured with a glucose oxidase method (Boehringer-Mannheim) on an LKB 7400 photometer. As the mean glucose concentrations had been higher in the original survey, a correction factor of 0.92 was used.

Serum insulin concentrations during the intravenous glucose tolerance test were measured in duplicate blood samples drawn before and four, six, eight, and 60 minutes after the start of the injection. The insulin index was defined as the ratio of the peak serum insulin concentration (mean of values at four, six, and eight minutes after injection) to the fasting serum insulin concentration. ${ }^{12}$ Insulin was measured with a commercially available kit (Phadebas Insulin Test, Pharmacia, Uppsala) on both occasions, but different standards were used. The values from the two investigations were therefore not directly comparable. Changes over time in hypertensive and control groups, however, were calculated and compared by using nontransformed data. For the description of changes in the hypertensive group values are presented as a percentage of the mean value in the control group at the follow up survey, which was taken as $100 \%$.

Serum cholesterol and triglyceride concentrations were measured on a type II Technicon autoanalyser ${ }^{18}$ both in samples from the follow up survey and in serum samples which had been stored in liquid nitrogen since the initial survey. Thus the analyses were by the same technique in the two surveys. The frozen samples were analysed at the same time as the samples from the follow up survey.

The cholesterol concentration in high density lipoprotein was assayed in the supernatant after precipitation of the very low and low density lipoproteins with heparin manganese chloride. ${ }^{19}$

\section{QUESTIONNAIRE}

A self administered medical questionnaire ${ }^{20}$ was used in the initial health survey to obtain familial and personal medical histories, including data on heritable factors, smoking, and physical activity. The same questions were asked in the follow up survey.

\section{STATISTICAL ANALYSIS}

An interaction test ${ }^{21}$ was applied for comparisons of changes between groups and between time points within groups. The model included factors for patient, group, time points, and group-time interaction. In one analysis diabetic state was used as an additional factor.

\section{Results}

The average length of the observation period was $9 \cdot 3$ (range 7-11) years for both groups (SD 0.73 for hypertensive men, 0.89 for controls). After nearly 10 years of treatment 79 men were still visiting the hypertension clinic. One died shortly before the reexamination date, one could not attend because of illness, and four failed to attend. Thus 73 hypertensive men were re-examined. Of 78 controls re-examined, 13 were taking thiazides or other blood pressure lowering drugs. Those receiving treatment were excluded from the analysis (none had diabetes); the final control group therefore comprised 65 men, none of whom had had diabetes when seen in 1970-3.

Five controls and two hypertensive men were receiv- 


\begin{tabular}{|c|c|c|c|c|c|c|c|c|c|}
\hline & \multirow[b]{2}{*}{ No treatment } & \multicolumn{2}{|c|}{ Single drug } & \multicolumn{5}{|c|}{ Combined treatment with $\beta$ blocker } & \multirow[b]{2}{*}{$\begin{array}{l}\text { Other drugs/ } \\
\text { combinations }\end{array}$} \\
\hline & & $\beta$ Blocker & Thiazide & Hydralazine & Thiazide & Spironolactone & $\begin{array}{c}\text { Hydralazine }+ \\
\text { thiazide }\end{array}$ & $\begin{array}{l}\text { Hydralazine }+ \\
\text { spironolactone }\end{array}$ & \\
\hline No of men & 3 & 13 & 3 & 6 & 18 & 3 & 14 & 4 & 9 \\
\hline
\end{tabular}

ing lipid lowering agents at re-examination. These men, along with those who had been given dietary advice during the follow up period (16 hypertensive men only), were also excluded from the final analysis when comparisons were made of metabolic changes over time; dietary treatment and hypolipidaemic drugs may affect serum lipid, glucose, and insulin values.

Treatment of hypertension-Propranolol was given as the first drug in previously untreated subjects. The next step, if required, was to add hydralazine, followed by thiazides if blood pressure was still not satisfactorily controlled. Propranolol was later changed to atenolol

TABLE II - Proportions of 73 hypertensive men receiving different drug treatments during observation period

\begin{tabular}{lcc}
\hline Drug (singly or in combination) & No $(\%)$ of men & Years of use \\
\hline Propranolol & $68(93)$ & 6.3 \\
Metoprolol or atenolol & $47(64)$ & $2 \cdot 6$ \\
Thiazides or loop diuretics & $58(79)$ & $5 \cdot 6$
\end{tabular}

TABLE III-Incidence of non-insulin-dependent (type II) diabetes during observation period among attenders at follow up survey (1980-3) stratified by diastolic blood pressure recorded at initial health survey $(1970-3)$

\begin{tabular}{|c|c|c|c|c|}
\hline & \multicolumn{4}{|c|}{$\begin{array}{c}\text { Diastolic blood pressure at initial survey } \\
(\mathrm{mm} \mathrm{Hg})\end{array}$} \\
\hline & $<85$ & $85-94$ & $95-104$ & $\geqslant 105$ \\
\hline $\begin{array}{l}\text { No of men } \\
\text { No }(\%) \text { who developed type II } \\
\text { diabetes }\end{array}$ & $\begin{array}{c}981 \\
25(2.5)\end{array}$ & $\begin{array}{c}631 \\
27(4 \cdot 3)\end{array}$ & $\begin{array}{c}91 \\
12(13 \cdot 1)\end{array}$ & $\begin{array}{c}93 \\
13(14 \cdot 0)\end{array}$ \\
\hline
\end{tabular}

*Table excludes 64 men who attended for follow up examination, of whom 38 had impaired glucose tolerance at follow up examination and 26 had diabetes at first examination 1970-3.

TABLE IV-Metabolic state of hypertensive and control groups at initial health survey (1970-3) and 95\% confidence intervals of estimated differences calculated from adjusted means between matched subjects

\begin{tabular}{|c|c|c|c|c|c|c|}
\hline & Group & $\begin{array}{c}\text { No of } \\
\text { men }\end{array}$ & Mean & SD & $\begin{array}{l}\text { Estimated } \\
\text { difference }\end{array}$ & $\begin{array}{l}95 \% \text { Confidence } \\
\text { interval of } \\
\text { estimated } \\
\text { difference }\end{array}$ \\
\hline \multicolumn{7}{|c|}{ Matched variables } \\
\hline Body mass index $\left(\mathrm{kg} / \mathrm{m}^{2}\right)$ & $\left\{\begin{array}{l}\text { Hypertensive } \\
\text { Control }\end{array}\right.$ & $\begin{array}{l}73 \\
65\end{array}$ & $\begin{array}{l}26 \cdot 92 \\
26 \cdot 50\end{array}$ & $\begin{array}{l}3 \cdot 47 \\
2.92\end{array}$ & $0 \cdot 37$ & $-1 \cdot 15$ to 1.89 \\
\hline Serum triglycerides $(\mathrm{mmol} / \mathrm{l})$ & $\left\{\begin{array}{l}\text { Hypertensive } \\
\text { Control }\end{array}\right.$ & $\begin{array}{l}73 \\
65\end{array}$ & $\begin{array}{l}2 \cdot 72 \\
2 \cdot 54\end{array}$ & $\begin{array}{l}1.47 \\
1.37\end{array}$ & $0 \cdot 17$ & -0.41 to 0.76 \\
\hline Serum cholesterol $(\mathrm{mmo} / \mathrm{l})$ & $\left\{\begin{array}{l}\text { Hypertensive } \\
\text { Control }\end{array}\right.$ & $\begin{array}{l}73 \\
65\end{array}$ & $\begin{array}{l}6 \cdot 82 \\
6 \cdot 58\end{array}$ & $\begin{array}{l}1.38 \\
1.05\end{array}$ & 0.24 & -0.24 to 0.71 \\
\hline $\begin{array}{l}\mathrm{k} \text { Value from intravenous glucose tolerance } \\
\text { test }\end{array}$ & $\left\{\begin{array}{l}\text { Hypertensive } \\
\text { Control }\end{array}\right.$ & $\begin{array}{l}51 \\
46\end{array}$ & $\begin{array}{l}1.55 \\
1.60\end{array}$ & $\left.\begin{array}{l}0.63 \\
0.66\end{array}\right\}$ & $-0 \cdot 10$ & -0.47 to 0.27 \\
\hline \multicolumn{7}{|c|}{ Non-matched variables } \\
\hline Body weight $(\mathbf{k g})$ & $\left\{\begin{array}{l}\text { Hypertensive } \\
\text { Control }\end{array}\right.$ & $\begin{array}{l}73 \\
65\end{array}$ & $\begin{array}{l}82 \cdot 48 \\
82 \cdot 40\end{array}$ & $\left.\begin{array}{r}11 \cdot 19 \\
9 \cdot 63\end{array}\right\}$ & 0.01 & $-5 \cdot 79$ to $5 \cdot 31$ \\
\hline Abdominal skinfold (mm) & $\left\{\begin{array}{l}\text { Hypertensive } \\
\text { Control }\end{array}\right.$ & 41 & $\begin{array}{l}26 \cdot 00 \\
23 \cdot 70\end{array}$ & $\left.\begin{array}{r}9 \cdot 10 \\
11 \cdot 30\end{array}\right\}$ & $0 \cdot 64$ & -8.04 to $9 \cdot 32$ \\
\hline Fasting blood glucose $(\mathrm{mmol} / \mathrm{l})$ & $\left\{\begin{array}{l}\text { Hypertensive } \\
\text { Control }\end{array}\right.$ & $\begin{array}{l}72 \\
65\end{array}$ & $\begin{array}{l}5 \cdot 23 \\
5 \cdot 14\end{array}$ & $\left.\begin{array}{l}0.79 \\
0.72\end{array}\right\}$ & 0.05 & -0.51 to 0.61 \\
\hline \multicolumn{7}{|l|}{ Serum insulin $(\mathrm{mU} / \mathrm{l})$ : } \\
\hline Fasting & $\left\{\begin{array}{l}\text { Hypertensive } \\
\text { Control }\end{array}\right.$ & $\begin{array}{l}54 \\
46\end{array}$ & $\begin{array}{l}18 \cdot 0 \\
15 \cdot 7\end{array}$ & $\begin{array}{r}8 \cdot 6 \\
11 \cdot 9\end{array}$ & $3 \cdot 0$ & 0.2 to 5.7 \\
\hline Peak value* & $\left\{\begin{array}{l}\text { Hypertensive } \\
\text { Control }\end{array}\right.$ & $\begin{array}{l}47 \\
44\end{array}$ & $\begin{array}{l}87 \cdot 5 \\
71 \cdot 0\end{array}$ & $\begin{array}{l}58 \cdot 6 \\
50 \cdot 1\end{array}$ & $22 \cdot 8$ & 1.3 to 44.3 \\
\hline $\begin{array}{l}\text { At } 60 \text { minutes in intravenous glucose } \\
\text { tolerance test }\end{array}$ & $\left\{\begin{array}{l}\text { Hypertensive } \\
\text { Control }\end{array}\right.$ & $\begin{array}{r}54 \\
46\end{array}$ & $\begin{array}{r}40 \cdot 5 \\
32 \cdot 9\end{array}$ & $\begin{array}{l}21 \cdot 1 \\
21 \cdot 5\end{array}$ & $9 \cdot 3$ & -0.0 to 18.6 \\
\hline Insulin indext & $\left\{\begin{array}{l}\text { Hypertensive } \\
\text { Control }\end{array}\right.$ & $\begin{array}{r}47 \\
44\end{array}$ & $\begin{array}{l}5.6 \\
5.6\end{array}$ & $\begin{array}{l}3.6 \\
4 \cdot 0\end{array}$ & 0.62 & -0.15 to 0.14 \\
\hline $\begin{array}{l}\text { High density lipoprotein cholesterol } \\
(\mathrm{mmo} / \mathrm{l})\end{array}$ & $\left\{\begin{array}{l}\text { Hypertensive } \\
\text { Control }\end{array}\right.$ & $\begin{array}{l}56 \\
49\end{array}$ & $\begin{array}{l}1 \cdot 10 \\
1 \cdot 10\end{array}$ & $\begin{array}{l}0.35 \\
0.28\end{array}$ & -0.01 & -0.01 to 0.95 \\
\hline
\end{tabular}

^Peak insulin value is mean of four, six, and eight minute insulin values after start of glucose injection. fInsulin index is ratio of peak insulin value divided by fasting serum insulin concentration. or metoprolol in two thirds of patients. Daily doses were in the following ranges: propranolol up to 1280 $\mathrm{mg}$ in the first years, then $80-320 \mathrm{mg}$; hydralazine 50 $200 \mathrm{mg}$; hydrochlorothiazide $12 \cdot 5-50 \mathrm{mg}$; atenolol and metoprolol $50-200 \mathrm{mg}$. Tables I and II give the numbers of patients taking single and combined doses.

Development of diabetes and impaired glucose tolerance - Twelve hypertensive men developed diabetes during the follow up period compared with two of the controls $(p<0.05)$. Two men in each group developed impaired glucose tolerance. Of all 2322 men who took part in the initial health survey, 1860 were re-examined in 1980-3. Of these, 353 men (19\%) were receiving treatment with antihypertensive drugs (191 (54\%) thiazides, 154 (44\%) non-selective $\beta$ blockers, 125 (35\%) selective $\beta$ blockers). Seventy seven of the 1860 men had developed non-insulin-dependent diabetes mellitus by the time of re-examination. The proportion who developed diabetes among the members of the cohort who were identified as hypertensive in $1970-3(18 / 141 ; 12.7 \%)$ was significantly higher than the proportion among the non-hypertensive population $(59 / 1655 ; 3.6 \%)$ $\left(\chi^{2}=24.6 ; p<0.001\right)$. None of the 20 hypertensive men who died before re-examination had diabetes recorded in their death certificates.

Heredity-Eighteen per cent of both study groups gave a history of diabetes among first degree relatives (13/73 hypertensive men, 12/65 controls). Four of the 12 hypertensive men who developed diabetes had such a family history.

Time of detection of diabetes-Of the 17 men who were already receiving antihypertensive treatment at the time of the first examination, three developed diabetes. Of the 56 with newly detected hypertension, nine developed diabetes. These proportions were not significantly different on $\chi^{2}$ testing. There was no evidence that detection and treatment of hypertension earlier than 50 years of age increased the risk of diabetes compared with detection and treatment at age 50.

Development of diabetes in different strata of blood pressure-In the whole cohort of 1860 men who attended follow up there was a positive relation between diastolic blood pressure at first examination and the subsequent development of diabetes (table III). The incidence of diabetes was seven times higher among men whose diastolic blood pressure had been $\geqslant 105 \mathrm{~mm} \mathrm{Hg}$ than among those in whom it had been $<85 \mathrm{~mm} \mathrm{Hg}$.

Anthropometric and metabolic characteristics-The matching procedure in the case-control study ensured that the mean values in the two groups were similar with respect to body mass index, $\mathrm{k}$ value, and serum triglyceride and cholesterol concentrations (table IV). Of the non-matched variables, abdominal skinfold thickness and fasting blood glucose, fasting serum insulin, and high density lipoprotein cholesterol concentrations also did not differ between the groups. The following variables were significantly different in the hypertensive men: blood pressure was higher (systolic $177 v 133 \mathrm{~mm} \mathrm{Hg}$, diastolic $111 v 82 \mathrm{~mm} \mathrm{Hg}$; both $\mathrm{p}<0.0001$ ); serum potassium concentration was lower $(3.9 v 4.0 \mathrm{mmol} / \mathrm{l} ; \mathrm{p}=0.013)$; peak serum insulin concentration was higher $(87.5 v 71.0 \mathrm{mU} / \mathrm{l} ; \mathrm{p}=0.04)$; serum insulin concentration at 60 minutes in the 
intravenous glucose tolerance test was higher $(40.5 v$ $32.9 \mathrm{mU} / \mathrm{l} ; \mathrm{p}=0.052$ ).

Changes in blood pressure, body weight, and metabolic variables over time-At follow up after 7-11 years blood pressure in the hypertensive group had decreased by $20 / 15 \mathrm{~mm} \mathrm{Hg}$ (table V), whereas in the control group it had increased by $14 / 8 \mathrm{~mm} \mathrm{Hg}$. Body weight had increased by $2.0 \mathrm{~kg}$ in the hypertensive group and 1.3 $\mathrm{kg}$ in the controls. The $\mathrm{k}$ value remained unaltered in the control group but had decreased in the hypertensive group from $1 \cdot 71$ to $1 \cdot 28(-34 \% ; p=0.015)$. This change, however, was not significantly different from the change in the controls (table V). These comparisons were made after excluding all men who had developed diabetes or who were receiving treatment

TABLE V-Blood pressure and metabolic variables at initial (first) health survey (1970-3) and at follow up (second) survey 7-11 years later, with 95\% confidence intervals of estimated differences within groups over time and between groups over time

\begin{tabular}{|c|c|c|c|c|c|c|c|}
\hline & & \multirow[b]{2}{*}{$\begin{array}{l}\text { No of } \\
\text { men }^{\star}\end{array}$} & \multirow[b]{2}{*}{ Mean } & \multirow[b]{2}{*}{ SD } & \multirow[b]{2}{*}{$\begin{array}{l}\text { Estimated } \\
\text { difference }\end{array}$} & \multicolumn{2}{|c|}{ 95\% Confidence interval of: } \\
\hline & & & & & & $\begin{array}{l}\text { Estimated } \\
\text { difference } \\
\text { within groups } \\
\text { over time }\end{array}$ & $\begin{array}{c}\text { Estimated } \\
\text { difference } \\
\text { between groups } \\
\text { over time }\end{array}$ \\
\hline \multicolumn{8}{|c|}{ Systolic blood pressure $(\mathrm{mm} \mathrm{Hg})$ : } \\
\hline $\begin{array}{l}\text { Hypertensive group } \\
\text { Control group }\end{array}$ & $\left\{\begin{array}{l}\text { 1st Survey } \\
\text { 2nd Survey } \\
\text { 1st Survey } \\
\text { 2nd Survey }\end{array}\right.$ & $\begin{array}{l}43 \\
43 \\
56 \\
56\end{array}$ & $\begin{array}{l}175 \cdot 4 \\
155 \cdot 0 \\
133 \cdot 1 \\
147 \cdot 2\end{array}$ & $\begin{array}{l}17 \cdot 1 \\
20 \cdot 8 \\
13 \cdot 2 \\
20 \cdot 5\end{array}$ & $\begin{array}{r}-20 \cdot 3 \\
14 \cdot 1\end{array}$ & $\begin{array}{c}-26 \cdot 1 \text { to }-14 \cdot 6 \\
9 \cdot 1 \text { to } 19 \cdot 2\end{array}$ & $-42 \cdot 1$ to $-26 \cdot 8$ \\
\hline \multicolumn{8}{|c|}{ Diastolic blood pressure ( $\mathrm{mm} \mathrm{Hg}$ ): } \\
\hline $\begin{array}{l}\text { Hypertensive group } \\
\text { Control group }\end{array}$ & $\begin{array}{l}\text { 1st Survey } \\
\text { 2nd Survey } \\
\text { 1st Survey } \\
\text { 2nd Survey }\end{array}$ & $\begin{array}{l}43 \\
43 \\
59 \\
59\end{array}$ & $\begin{array}{r}110 \cdot 9 \\
96 \cdot 2 \\
81 \cdot 8 \\
89 \cdot 3\end{array}$ & $\begin{array}{l}8 \cdot 5 \\
7 \cdot 9 \\
8 \cdot 9 \\
9 \cdot 2\end{array}$ & $\begin{array}{r}-14 \cdot 8 \\
7 \cdot 5\end{array}$ & $\begin{array}{c}-17 \cdot 5 \text { to }-12 \cdot 0 \\
5 \cdot 1 \text { to } 9 \cdot 9\end{array}$ & $-25 \cdot 9$ to $-18 \cdot 6$ \\
\hline \multicolumn{8}{|l|}{ Body weight $(\mathbf{k g})$ : } \\
\hline $\begin{array}{l}\text { Hypertensive group } \\
\text { Control group }\end{array}$ & $\left\{\begin{array}{l}\text { 1st Survey } \\
\text { 2nd Survey } \\
\text { 1st Survey } \\
\text { 2nd Survey }\end{array}\right.$ & $\begin{array}{l}43 \\
43 \\
59 \\
59\end{array}$ & $\begin{array}{l}80 \cdot 23 \\
82 \cdot 27 \\
82 \cdot 07 \\
82 \cdot 81\end{array}$ & $\begin{array}{l}11 \cdot 21 \\
11 \cdot 62 \\
10.10 \\
11 \cdot 38\end{array}$ & $\begin{array}{l}2 \cdot 04 \\
1 \cdot 29\end{array}$ & $\begin{array}{r}0.52 \text { to } 3.55 \\
-0.58 \text { to } 2.07\end{array}$ & -0.72 to 3.30 \\
\hline \multicolumn{8}{|c|}{ Abdominal skinfold (mm): } \\
\hline $\begin{array}{l}\text { Hypertensive group } \\
\text { Control group }\end{array}$ & $\left\{\begin{array}{l}\text { 1st Survey } \\
\text { 2nd Survey } \\
\text { 1st Survey } \\
\text { 2nd Survey }\end{array}\right.$ & $\begin{array}{l}22 \\
43 \\
37 \\
56\end{array}$ & $\begin{array}{l}23.69 \\
31 \cdot 63 \\
22 \cdot 25 \\
29 \cdot 79\end{array}$ & $\begin{array}{r}8 \cdot 48 \\
8 \cdot 52 \\
10 \cdot 72 \\
10 \cdot 63\end{array}$ & 7.56 & $\begin{array}{l}3.48 \text { to } 11.66 \\
4.15 \text { to } 10.47\end{array}$ & $-4 \cdot 91$ to $5 \cdot 43$ \\
\hline \multicolumn{8}{|c|}{ Fasting blood glucose $(\mathrm{mmol} / \mathrm{l})$ : } \\
\hline $\begin{array}{l}\text { Hypertensive group } \\
\text { Control group }\end{array}$ & $\left\{\begin{array}{l}\text { 1st Survey } \\
\text { 2nd Survey } \\
\text { 1st Survey } \\
\text { 2nd Survey }\end{array}\right.$ & $\begin{array}{l}43 \\
42 \\
56 \\
56\end{array}$ & $\begin{array}{l}5 \cdot 04 \\
5 \cdot 16 \\
5 \cdot 06 \\
5 \cdot 02\end{array}$ & $\begin{array}{l}0.62 \\
0.46 \\
0.56 \\
0.49\end{array}$ & $\begin{array}{r}0.14 \\
-0.03\end{array}$ & $\begin{array}{l}-0.02 \text { to } 0.31 \\
-0 \cdot 18 \text { to } 0 \cdot 10\end{array}$ & -0.04 to 0.40 \\
\hline \multicolumn{8}{|c|}{ k Value from intravenous glucose tolerance test: } \\
\hline $\begin{array}{l}\text { Hypertensive group } \\
\text { Control group }\end{array}$ & $\begin{array}{l}\text { Ist Survey } \\
\text { 2nd Survey } \\
\text { 1st Survey } \\
\text { 2nd Survey }\end{array}$ & $\begin{array}{l}29 \\
42 \\
38 \\
54\end{array}$ & $\begin{array}{l}1 \cdot / 1 \\
1 \cdot 28 \\
1.63 \\
1.64\end{array}$ & $\begin{array}{l}0.60 \\
0.52 \\
0.66 \\
0.86\end{array}$ & $\begin{array}{r}-0.35 \\
0.09\end{array}$ & $\begin{array}{l}-6.63 \text { to }-0.07 \\
-0.34 \text { to } 0.15\end{array}$ & -0.63 to $0 \cdot 11$ \\
\hline \multicolumn{8}{|c|}{ Serum triglycerides $(\mathrm{mmol} / \mathrm{l})$} \\
\hline $\begin{array}{l}\text { Hypertensive group } \\
\text { Control group }\end{array}$ & $\left\{\begin{array}{l}\text { 1st Survey } \\
\text { 2nd Survey } \\
\text { 1st Survey } \\
\text { 2nd Survey }\end{array}\right.$ & $\begin{array}{l}43 \\
41 \\
56 \\
50\end{array}$ & $\begin{array}{l}2 \cdot 29 \\
2 \cdot 61 \\
2 \cdot 45 \\
2 \cdot 04\end{array}$ & $\begin{array}{l}0 \cdot 85 \\
1 \cdot 41 \\
1 \cdot 39 \\
1 \cdot 11\end{array}$ & $\begin{array}{r}0.32 \\
-0.32\end{array}$ & $\begin{array}{l}-0.02 \text { to } 0.67 \\
-0.72 \text { to }-0.10\end{array}$ & $0 \cdot 29$ to $1 \cdot 17$ \\
\hline \multicolumn{8}{|c|}{ Serum cholesterol (mmol/l): } \\
\hline $\begin{array}{l}\text { Hypertensive group } \\
\text { Control group }\end{array}$ & $\left\{\begin{array}{l}\text { lst Survey } \\
\text { 2nd Survey } \\
\text { 1st Survey } \\
\text { 2nd Survey }\end{array}\right.$ & $\begin{array}{l}43 \\
41 \\
56 \\
50\end{array}$ & $\begin{array}{l}6 \cdot 76 \\
6 \cdot 42 \\
6 \cdot 62 \\
6 \cdot 18\end{array}$ & $\begin{array}{l}1 \cdot 50 \\
1 \cdot 11 \\
1.07 \\
0.98\end{array}$ & $\begin{array}{l}-0.32 \\
-0.32\end{array}$ & $\begin{array}{l}-0.65 \text { to }-0.00 \\
-0.62 \text { to }-0.04\end{array}$ & -0.43 to 0.44 \\
\hline \multicolumn{8}{|c|}{ High density lipoprotein cholesterol $(\mathrm{mmol} / \mathrm{l})$ : } \\
\hline $\begin{array}{l}\text { Hypertensive group } \\
\text { Control group }\end{array}$ & $\left\{\begin{array}{l}\text { lst Survey } \\
\text { 2nd Survey } \\
\text { 1st Survey } \\
\text { 2nd Survey }\end{array}\right.$ & $\begin{array}{l}34 \\
40 \\
43 \\
54\end{array}$ & $\begin{array}{l}1 \cdot 11 \\
1 \cdot 03 \\
1 \cdot 11 \\
1 \cdot 08\end{array}$ & $\begin{array}{l}0 \cdot 40 \\
0 \cdot 23 \\
0 \cdot 27 \\
0 \cdot 34\end{array}$ & $\begin{array}{l}-0 \cdot 12 \\
-0 \cdot 03\end{array}$ & $\begin{array}{l}-0.24 \text { to } 0.00 \\
-0.14 \text { to } 0.08\end{array}$ & -0.26 to 0.07 \\
\hline
\end{tabular}

*Table excludes hypertensive men and controls with diabetes or who had received dietary advice or were taking lipid lowering drugs at follow up.

TABLE VI-Relative percentage values of fasting and peak serum insulin concentrations at initial (first) and follow up (second) health surveys in hypertensive men who developed diabetes mellitus or impaired glucose tolerance during observation period and those who did not. Values in controls who did not develop these disorders taken as $100 \%$

\begin{tabular}{|c|c|c|c|c|}
\hline & & $\begin{array}{c}\text { Hypertensive men } \\
\text { who developed } \\
\text { diabetes or impaired } \\
\text { glucose tolerance } \\
(n=14)\end{array}$ & $\begin{array}{c}\text { Hypertensive men } \\
\text { who did not develop } \\
\text { diabetes or impaired } \\
\text { glucose tolerance } \\
(\mathbf{n}=59)\end{array}$ & $\begin{array}{l}\text { Controls who did not } \\
\text { develop diabetes or } \\
\text { impaired glucose } \\
\text { tolerance } \\
(\mathbf{n}=61)^{\star}\end{array}$ \\
\hline $\begin{array}{l}\text { Relative value of fasting } \\
\text { serum insulin }(\%) \dagger \\
\text { Relative value of peak serum } \\
\text { insulin }(\%) \ddagger\end{array}$ & $\left\{\begin{array}{l}\text { lst Survey } \\
\text { 2nd Survey } \\
\text { lst Survey } \\
\text { 2nd Survey }\end{array}\right.$ & $\begin{array}{r}178 \\
205 \\
113 \\
62\end{array}$ & $\begin{array}{l}103 \\
146 \\
134 \\
134\end{array}$ & $\begin{array}{l}100 \\
100 \\
100 \\
100\end{array}$ \\
\hline
\end{tabular}

\#Values in controls assumed to be unchanged over time. Adjustment to $100 \%$ done after interaction test. †Values corrected from second survey.

$\ddagger$ Insulin peak in follow up survey multiplied by 2.153 and transformed into percentage scale where $70.4 \mathrm{mU} / \mathrm{l}=$ $100 \%$ with lipid lowering drugs or diet in 1980-3. Serum triglyceride concentration had declined in the control group $(2.45$ to $2.04 \mathrm{mmol} / \mathrm{l})$ and increased in the hypertensive group $(2.29$ to $2.61 \mathrm{mmol} / \mathrm{l})$. These changes were significantly different between the groups (table V). Changes in serum cholesterol and high density lipoprotein cholesterol concentrations over time showed no difference between the groups. These findings were consistent whether or not men receiving lipid lowering agents or diet were excluded.

Comparison of men who developed diabetes with those who did not-Review of values obtained at the initial examination showed that the hypertensive men who later developed diabetes (12) or impaired glucose tolerance (two) could be distinguished from the remaining hypertensive men in six main ways: $(a)$ their fasting serum insulin concentration was higher $(26 \cdot 1 v$ $15.2 \mathrm{mU} / 1(\mathrm{p}<0.0001)$ or $178 \% v 103 \%$ of the control value $(\mathrm{p}<0.0001)$ ) (table VI); $(b)$ their peak serum insulin concentration was lower $(78.9 v 94.3 \mathrm{mU} / \mathrm{l}(\mathrm{p}=$ 0.067 ) or $113 \% v 134 \%$ of the control value) (table VI); (c) their insulin index was lower $(3.4 v 6.6 ; 95 \%$ confidence interval of difference 1.4 to 5.4$)$; (d) their serum triglyceride concentration was higher $(3.43 v$ $2.56 \mathrm{mmol} / \mathrm{l} ; 95 \%$ confidence interval of difference -1.51 to -0.27$) ;(e)$ their body mass index was higher (28.88 $v 26.41 ; 95 \%$ confidence interval of difference -3.10 to -1.58$)$; and $(f)$ their $\mathrm{k}$ value was lower $(1.29 v$ $1.68 ; 95 \%$ confidence interval of difference -0.02 to 0.68 ) (table VII). The four men in the control group who developed diabetes or impaired glucose tolerance also had higher fasting serum insulin concentrations than the remainder of the control group at the initial examination $(21.5 v 14.7 \mathrm{mU} / \mathrm{l})$. The small number precluded statistical analysis. There was no difference in fasting blood glucose and serum cholesterol concentrations between those who developed diabetes and those who did not.

Relative changes in serum insulin concentrations during treatment for hypertension - To evaluate the influence of long term antihypertensive treatment on metabolic variables with no interference from diabetes or impaired glucose tolerance we compared the changes over time between non-diabetic men in the hypertensive and control groups. Excluding men who had received hypolipidaemic drugs or dietary advice did not alter the results and they were therefore included in the analysis (table VII). The fasting serum insulin concentration increased in non-diabetic hypertensives compared with non-diabetic controls (table VI), but not significantly. There was no change with time in peak serum insulin concentration in non-diabetic hypertensives relative to controls, though the value in diabetic hypertensives showed a substantial fall $(\mathbf{p}<0.0001)$ (table VI). The insulin index (table VII) decreased with time in both non-diabetic and diabetic hypertensives compared with non-diabetic controls.

\section{Discussion}

The incidence of diabetes mellitus among people with untreated hypertension is not known. In this study, however, the hypertensive men who later developed diabetes differed from those who did not with respect to metabolic variables and showed signs of disturbed glucose metabolism at around the age of 50 , many years before the onset of diabetes. Thus they had a higher fasting serum insulin concentration and lower peak serum insulin concentration $(p=0.064)$, giving a lower insulin index at the initial examination. They also had a higher body mass index and serum triglyceride concentration and a lower $(p=0.065)$, but still normal, $\mathrm{k}$ value compared with those hypertensive men who did not develop diabetes. Several of these signs-for example, a high fasting serum insulin 


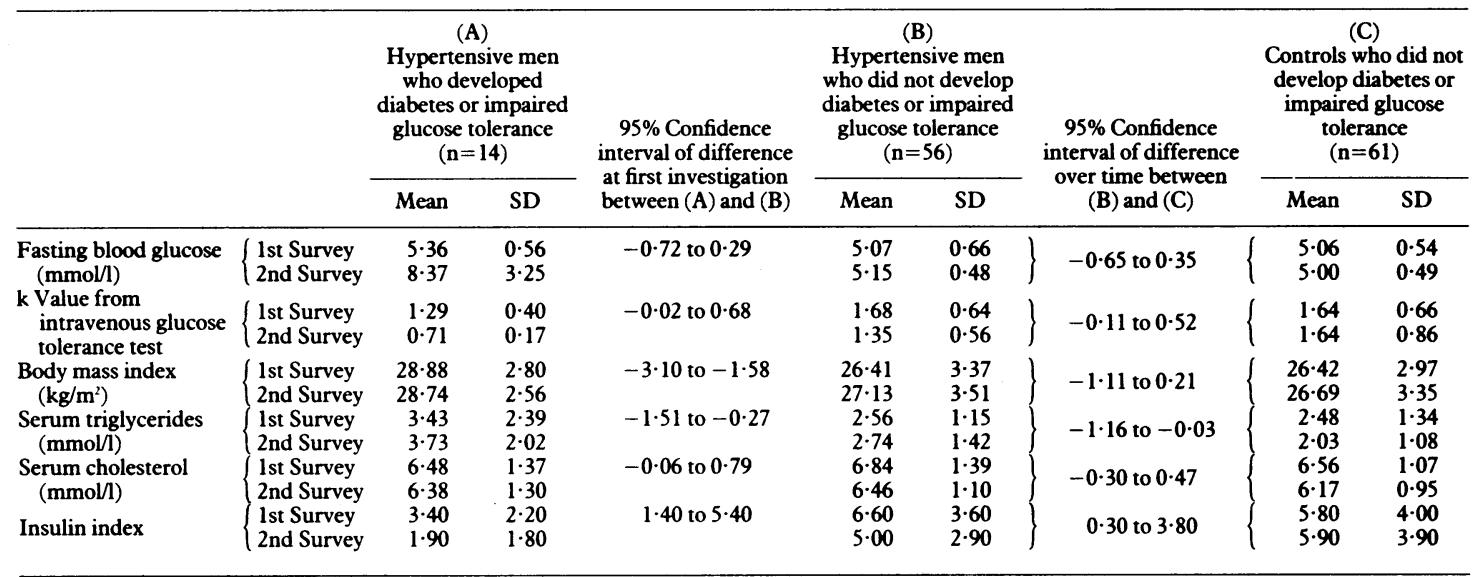

concentration and an increased serum triglyceride concentration-indicate increased insensitivity to insulin. The sensitivity to insulin as measured by the hyperinsulinaemic euglycaemic clamp technique is reduced in the hypertensive state even after correction for a higher body mass index. Reduced sensitivity to insulin may therefore be a primary state in people with hypertension. Furthermore, a low insulin index has been regarded as an early prognostic sign of the later development of non-insulin-dependent (type II) diabetes mellitus. ${ }^{22}$

In our subgroup of treated hypertensive men who did not develop diabetes the insulin index and serum triglyceride concentration changed in a different way from that in the control group despite the two groups having been similar at the start. That the changes were different in the hypertensive men supports but does not prove the hypothesis that the changes were the effects of long term treatment. It may be a feature of hypertensive men that insulin sensitivity worsens with time. This worsening, however, is probably not related to the hypertensive state itself, as the blood pressure decreased in the hypertensive group and increased in the controls, and these changes should have resulted in the opposite alterations in insulin values to those that were actually found. These data therefore offer some support to the view that today's first line antihypertensive drugs may cause a decrease in insulin sensitivity which does not disappear with time and may elicit the development of diabetes mellitus, particularly in men already predisposed to the disease.

There are conflicting data on whether the effects of drugs on lipoprotein metabolism disappear with time. In one study, for example, the changes in serum lipid values detected after six months of treatment with chlorthalidone had vanished after two years of treatment. ${ }^{23}$ By contrast, in the multiple risk factor intervention trial dietary treatment of hyperlipidaemia reversed the changes induced by antihypertensive drugs but the plasma lipid values did not reach as low as in a comparison group that was also given dietary treatment but no antihypertensive drugs. Thus antihypertensive pharmacotherapy counteracted dietary treatment so that the hypertensive group still had higher serum triglyceride and lower high density lipoprotein cholesterol concentrations than the comparison group six years after the start of treatment. ${ }^{11}$ In our study even after treatment for almost 10 years the change in serum triglyceride values over time differed significantly between the hypertensive and control groups. Serum triglyceride concentrations increased among non-diabetic hypertensive men but decreased among non-diabetic controls $(\mathrm{p}<0.02)$.

The risk factor pattern for coronary heart disease in hypertensive men is improved by antihypertensive treatment in so far as the blood pressure is reduced. But the increased risk of coronary heart disease associated with raised blood pressure may not depend on the blood pressure alone. High blood pressure is only one aspect of a metabolic syndrome which is also characterised by a raised serum insulin concentration and increased serum triglyceride values, and all these aspects may be related to decreased insulin sensitivity. Possibly the other characteristics of the syndrome may have been underestimated as risk factors for coronary heart disease. Increased serum insulin and triglyceride concentrations have been found to be risk factors in prospective epidemiological studies. ${ }^{2428}$ If antihypertensive treatment as used in this study is associated with a deterioration of the other features of the metabolic syndrome such changes may partly explain the failure of many studies on treatment of hypertension to reduce the incidence of coronary heart disease.

This work was supported by grants from the Swedish Diabetic Association and the Swedish Medical Research Council (5446), the Swedish National Association Against Heart and Lung Diseases, the King Gustaf V Research Foundation, and the Swedish Society of Physicians.

1 Anonymous. Risks of antihypertensive therapy [Editorial]. Lancet 1986;ii: 1075-6.

2 Bengtsson C, Blohmé G, Lapidus L. Do antihypertensive drugs precipitate diabetes? Br Med f 1984;289:1495-7.

3 Carliner NH, Schelling JL, Pattersson RR, Okun R, Davis M. Thiazide- and phatalamidine-induced hyperglycemia in hypertensive patients. $\mathcal{J} A M A$ 1965;7:535-40.

4 Breckenridge A, Dollery CT, Welborn TA, Fraser R. Glucose tolerance in hypertensive patients on long-term diuretic treatment. Lancet 1967; i:61-4.

5 Murphy MB, Lewis PJ, Kohner E, Schumer B, Dollery CT. Glucose intolerance in hypertensive patients treated with diuretics; a fourteen-year follow-up. Lancet 1982;ii:1293-5.

6 Ames RP, Hill P. Increase in serum-lipids during treatment of hypertension with chlorthalidone. Lancet 1976; i:721-3.

7 Medical Research Council Working Party on Mild to Moderate Hypertension. Adverse reactions to bendrofluazide and propranolol for the treatment of mild hypertension. Lancet 1981;ii:539-43.

8 Tanaka N, Sakaguchi S, Oshige K, Niimura T, Kanehisa T. Effect of chronic administration of propranolol on lipoprotein composition. Metabolism 1976;25:1071-5.

9 Waal-Manning HJ, Sim

BrMed f 1977;ii:705.

10 Day JL, Simpson N, Metcalfe J, Page RL. Metabolic consequences of atenolol and propranolol in treatment of essential hypertension. $\mathrm{Br} \mathrm{Med} \mathcal{J}$ 1979; i:7780.

11 Lasser NL, Grandits G, Caggiula AW, et al. Effects of antihypertensive therapy on plasma lipids and lipoproteins in the multiple risk factor intervention trial. $A m \mathcal{F}$ Med 1984;76(2A):52-66.

12 Hedstrand $\mathrm{H}$. A study of middle-aged men with particular reference to risk factors for cardiovascular disease. Ups $\mathcal{F}$ Med Sci 1975;suppl 19. (Thesis.)

13 Áberg H, Hedstrand H, Lithell H. Blood pressure control in a middle-aged male population. A 6-9 year follow-up with special reference to the problem of non-responders. Acta Med Scand 1980;208:467-71.

14 Berglund G, Larsson B, Andersson O, et al. Body composition and glucose metabolism in hypertensive middle-aged males. Acta Med Scand 1976;200: $163-9$.

15 Edwards DA, Hammond WH, Healy MJ, Tanner JM, Whitehouse RH. Design and accuracy of calipers for measuring subcutaneous tissue thickness. Design and accuracy of calip
$B r \mathcal{F}$ Nutr 1955;9:133-43.

16 Ikkos D, Luft R. On the glucose tolerance test. Acta Endocrinol 1957;25:31234.

17 National Diabetes Data Group. Classification and diagnosis of diabetes mellitus and other categories of glucose intolerance. Diabetes 1979; 28: 103957. 
18 Rush RL, Leon L, Turrel J. Automated simultaneous cholesterol and triglyceride determination on the Auto-Analyzer II instrument. Advances in
automated analysis. Vol 1. Miami, Florida: Thurman Associates, 1971:503-7.

19 Burstein M, Samaille J. Sur un dosage rapide de cholésterol lié au alpha- et bétalipoprotéins du sérum. Clin Chim Acta 1955;5:609.

20 Collen MF, Cutler JL, Siegelaub AB, Cella RL. Reliability of a selfadministered medical questionnaire. Arch Intern Med 1969;123:664-71.

21 Brownlee KA. Statistical theory and methodology in science and engineering. New York: Wiley, 1965

22 Cerasi $E$, Luft $R$. The plasma insulin response to glucose infusion in healthy subjects and in diabetes mellitus. Acta Endocrinol 1967;55:278-304.

23 Hulley SB, Furberg CD, Gurland B, et al. Systolic hypertension in the elderly program (SHEP): antihypertensive efficacy of chlorthalidone. $A m \mathcal{f}$ Cardiol 1985;56:913-20.

24 Castelli WP. The triglyceride issue: a view from Framingham. Am Heart $f$ 1986;112:432-7.
25 Åberg H, Lithell H, Selinus I, Hedstrand H. Serum triglycerides are a risk factor for myocardial infarction but not for angina pectoris. Results from 10-year follow-up of Uppsala primary preventive study. Atherosclerosis 1985;54:89-97.

26 Pyörälä K. Relationship of glucose tolerance and plasma insulin to the incidence of coronary heart disease: results from two population studies in Finland. Diabetes Care 1979;2:131-41.

27 Ducimetiere P, Eschwege E, Papoz L, Richard JL, Claude JR, Rosselin G. Relationship of plasma insulin levels to the incidence of myocardial infarction and coronary heart disease mortality in a middle-aged population Diabetologia 1980;19:205-10.

28 Welborn TA, Wearne K. Coronary heart disease incidence and cardiovascula mortality in Busselton with reference to glucose and insulin concentrations. Diabetes Care 1979;2:154-60.

(Accepted 3 February 1989)

\title{
Sensitivity to insulin during treatment with atenolol and metoprolol: a randomised, double blind study of effects on carbohydrate and lipoprotein metabolism in hypertensive patients
}

\author{
Thomas Pollare, Hans Lithell, Ingemar Selinus, Christian Berne
}

\section{Abstract}

Objective-To compare the effects of metoprolol and atenolol on carbohydrate and lipid metabolism and on insulin response to an intravenous glucose load.

Design-Randomised, double blind, double dummy, controlled crossover trial.

Setting - University Hospital, Uppsala, Sweden.

Patients -60 Patients with primary hypertension (diastolic blood pressure when resting supine 95-119 $\mathrm{mm} \mathrm{Hg}$ on at least two occasions during four to six weeks of treatment with placebo) randomised to receive either metoprolol $(n=30)$ or atenolol $(n=30)$ during the first treatment period.

Interventions-Placebo was given for a run in period of four to six weeks. Metoprolol $100 \mathrm{mg}$ twice daily or atenolol $25 \mathrm{mg}$ twice daily was then given for 16 weeks. The two drugs were then exchanged and treatment continued for a further 16 weeks.

End point-Evaluation of effects of treatment with metoprolol and atenolol on glucose, insulin, and lipid metabolism and glucose disposal mediated by insulin.

Measurements and main results-Reduction of blood pressure was similar and satisfactory during treatment with both drugs. Glucose uptake mediated by insulin was measured during a euglycaemic hyperinsulinaemic clamp to evaluate patients' sensitivity to insulin. Glucose uptake decreased from 5.6 to 4.5 $\mathrm{mg} / \mathrm{kg} / \mathrm{min}$ when patients were taking metoprolol and from 5.6 to $4.9 \mathrm{mg} / \mathrm{kg} / \mathrm{min}$ when they were taking atenolol. Both.drugs caused a small increase in fasting plasma insulin and blood glucose concentrations and glycated haemoglobin concentration. Despite decreased sensitivity to insulin the increase in insulin concentration in response to an intravenous glucose tolerance test was small, suggesting inhibition of release of insulin. Very low density lipoprotein and low density lipoprotein triglyceride concentrations were increased with both drugs and high density lipoprotein cholesterol concentration was decreased. Low density lipoprotein cholesterol concentration was not affected.

Conclusions-Long term use of metoprolol and atenolol causes metabolic abnormalities that may be related to the increased incidence of diabetes in patients with hypertension who are treated pharmacologically. These results may help to explain why the two drugs have failed consistently to reduce the incidence of coronary heart disease in several large scale studies.

\section{Introduction}

During the past 20 years there has been much emphasis on detecting and treating hypertension, which is an important risk factor for cardiovascular disease, ${ }^{1}$ and several large scale trials have shown that a reduction in blood pressure is associated with a decrease in cardiovascular morbidity and mortality. ${ }^{2.5}$ Some of the drugs used to treat hypertension, however, have adverse effects, including disturbances of serum lipid concentrations and glucose metabolism. ${ }^{5.7}$ Treatment with thiazide diuretics and $\beta$ blockers has been associated with an increased incidence of impaired glucose tolerance ${ }^{6}$ and diabetes. ${ }^{57}$ Few attempts have been made, however, to evaluate specific influences of pharmacological treatment of hypertension on glucose metabolism-for example, to determine whether it decreases secretion of insulin or sensitivity to insulin, or both. Sensitivity, to insulin may be important as studies have shown that hypertension is accompanied by resistance to insulin..$^{8-10}$

We evaluated the effect of long term treatment with two widely used $\beta_{1}$ adrenergic blockers, metoprolol and atenolol, on glucose disposal mediated by insulin and examined their effects on glucose, insulin, and lipid metabolism.

\section{Patients and methods}

\section{CRITERIA FOR INCLUSION}

Patients were recruited from a health screening survey in Uppsala, Sweden. All had primary hypertension, defined as a stable diastolic blood pressure when resting supine of $95-119 \mathrm{~mm} \mathrm{Hg}$ on at least two occasions during four to six weeks of treatment with placebo. Any current antihypertensive treatment was stopped and a placebo was given single blind for four to six weeks. Patients with newly detected hypertension were followed up for three to four months to make sure that their raised blood pressure was stable before they entered the single blind placebo period.

\section{CRITERIA FOR EXCLUSION}

Criteria for exclusion were: clinical or laboratory evidence of hepatic or renal disease, obstructive pulmonary disease, Raynaud's disease, or thyroid dys-

Pollare. 